\title{
The Technology Improvement on Production of
}

\section{the Polycrystalline Silicon by Zinc Production}

\author{
Wang Shuya \\ Qinghai institute of salt lake \\ Chinese academy of sciences \\ Xining, China \\ Qi Mixiang \\ Qinghai institute of salt lake \\ Chinese academy of sciences \\ Xining, China
}

\author{
Li Chunsong \\ New energy branch \\ Qinghai Huanghe hydropower \\ development CO.LTD \\ Xining, China \\ Yang Zhanshou \\ Qinghai institute of salt lake \\ Chinese academy of sciences \\ Xining, China
}

\author{
Wang Shuxuan \\ Qinghai institute of salt lake \\ Chinese academy of sciences \\ Xining, China \\ Zou Xingwu \\ Qinghai institute of salt lake \\ Chinese academy of sciences \\ Xining, China
}

\begin{abstract}
This report details the thermodynamic analysis on zinc reduction of silicon tetrachloride. The diagrams of $\Delta_{r} G_{m}^{\Theta}-T$ and $K_{p}^{\Theta}$-T for the Zinc production reaction were plotted. The reasonable temperature range was obtained by the thermodynamic analysis. The theoretical basis of improvements of the equipment and process flow were provided by the thermodynamic analysis. The continuous processing, low Energy consumption and the shortening of process flow were the advantage in the improvement of equipment and process.
\end{abstract}

Keywords-zinc reduction; polycrystalline silicon; Process; energy consumption

\section{INTRODUCTION}

Clean energy sources are increasingly attractive due to the awareness of global warming and environmental pollution. Therefore, photovoltaic cells are expected to contribute to a solution to global warming and environmental problems [1]. In recent years, with the rapid development of photovoltaic cells and polycrystalline silicon industry, a large quantity of industrial by-product silicon tetrachloride exists in the polycrystalline silicon production industry[2].

Polycrystalline silicon is used as an important raw material for solar cell production. Improved Siemens method was widely used by polycrystalline silicon manufacturers at home and abroad. Although the Siemens method has been well developed and is essential for highly purified silicon production for the semiconductor industries, this technique has several problems, such as high cost facilities and low silicon production yield. A ton polycrystalline silicon can output $10 \sim$ 15 tons silicon tetrachloride with improved Siemens method. The low recovery of silicon tetrachloride was the main reason for the high cost. The majority of polycrystalline silicon manufacturers face the pressure that production costs is higher than the product selling price.
However, when enterprises are facing pressure to survive, innovation is sometimes necessary means. We decided to investigate the zinc reduction method. Zinc reduction method was not a new method [3]. However, how to improve the equipment and process flow are problems that are need further researches. Based on the thermodynamic analysis, this paper researches the improvements of the equipment and process flow.

\section{THERMODYNAMIC ANALYSIS}

A. Thermodynamic Data

The overall reaction is,

$$
2 \mathrm{Zn}(\mathrm{g})+\mathrm{SiCl}_{4}(\mathrm{~g}) \rightarrow \mathrm{Si}(\mathrm{s})+2 \mathrm{ZnCl}_{2}(\mathrm{~g}) .
$$

The thermodynamic data used for the estimation of reaction yields was shown in Table 1. The computational formula was shown in (2)-(5)[4].

$$
\begin{aligned}
& \Delta_{r} H_{m}^{\Theta}=\sum_{B} v_{B}^{\Delta} f_{m}^{\Theta} \\
& \Delta_{r} S_{\mathrm{m}}^{\Theta}=\sum_{B} v_{B} S_{m}^{\Theta} \\
& \Delta_{r} G_{m}^{\Theta}(T)=\Delta_{r} H_{\mathrm{m}, 298.15 \mathrm{~K}}^{\Theta}+\underset{298.15}{j_{j}} \Delta C_{p, m} d T-T \Delta_{r} S_{\mathrm{m}, 298.15 \mathrm{~K}}^{\Theta}-T \underset{298.15}{\int_{j}^{K}} \frac{\Delta C_{p, m}}{T} d T . \\
& \Delta_{r} G_{m}^{\Theta}(K)=-R T \ln K_{p}^{\Theta}
\end{aligned}
$$




\section{B. Graphic Analyses}

The diagrams of $\Delta_{r} G_{m}^{\Theta}-\mathrm{T}$ and $K_{p}^{\Theta}$-T for the Zinc production reaction were plotted in Fig.1 and Fig.2.

TABLE I. THE THERMODYNAMIC DATA USED FOR THE ESTIMATION OF REACTION YIELDS[5]-[7]

\begin{tabular}{|c|l|l|l|c|c|}
\hline \multirow{2}{*}{$\begin{array}{c}\text { Chemical } \\
\text { species }\end{array}$} & \multicolumn{5}{|c|}{ Thermodynamics Parameter } \\
\cline { 2 - 6 } & $\Delta_{f} H_{\mathrm{m}, 298.15 \mathrm{~K}}^{\Theta}$ & $S_{m, 298.15 \mathrm{~K}}^{\Theta}$ & \multicolumn{3}{|c|}{$\boldsymbol{C}_{\boldsymbol{p}, \boldsymbol{m}}$} \\
\cline { 4 - 7 } & $\boldsymbol{K J} \cdot \mathbf{m o l}^{\mathbf{1}}$ & $\boldsymbol{J} \cdot \mathbf{m o l}^{\mathbf{1}} \cdot \boldsymbol{K}^{\mathbf{1}}$ & $\boldsymbol{a}$ & $\boldsymbol{b}$ & $\boldsymbol{c}$ \\
\hline $\mathrm{SiCl}_{4}(\mathrm{~g})$ & -662.7 & 330.8 & 101.5 & 6.9 & 11.5 \\
\hline $\mathrm{Zn}(\mathrm{g})$ & 130.4 & 161.0 & 20.79 & & \\
\hline $\mathrm{Si}(\mathrm{s})$ & 0.0 & 18.8 & 22.82 & 3.9 & -3.5 \\
\hline $\mathrm{ZnCl}_{2}(\mathrm{~g})$ & -248.6 & 276.1 & 60.25 & 0.8 & \\
\hline
\end{tabular}

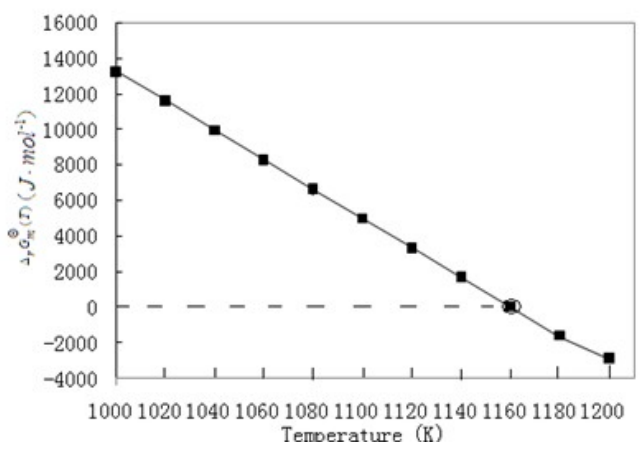

Figure 1. The diagrams of $\Delta_{r} G_{m}^{\Theta}-\mathrm{T}$

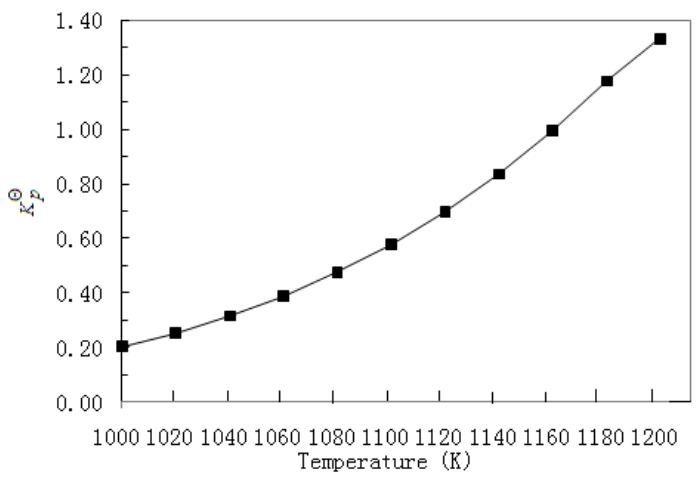

Figure 2. The diagram of $K_{p}^{\Theta}-\mathrm{T}$

The theoretical reaction temperature of (1) is about $1160 \mathrm{~K}$, as shown in Fig.1. $K_{p}^{\Theta}$ decreased in Accordance with temperature. The speed increases quickly when the temperature is above $1120 \mathrm{~K}$. After the temperature reaches $1160 \mathrm{~K}$, the $K_{p}^{\Theta}$ will exceed 1 . The rational temperature range is $1120 \mathrm{~K}$ $1200 \mathrm{~K}$.

\section{IMPROVEMENTS OF THE EQUIPMENT AND PROCESS FLOW}

\section{A. Improvements of the Process Flow}

Fig. 3 was the existing process flow. The improved Process

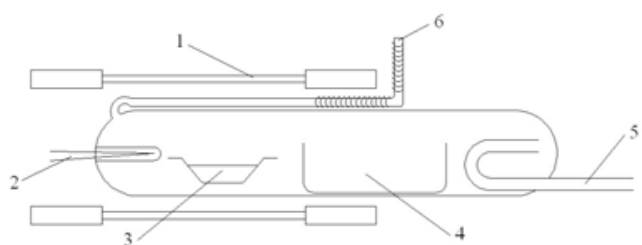

1- Heating installation 2-Thermocouple $3-\mathrm{Zn}(\mathrm{s})$

4- Collection device 5-Vent 6- Silicon tetrachloride inlet

Figure 3. The existing precess flow[8]

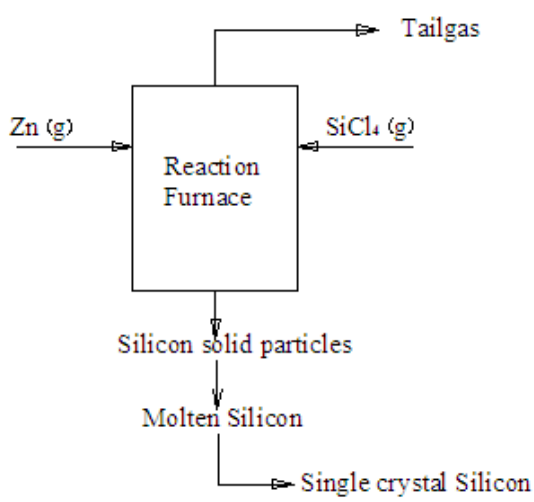

Figure 4. The improved Process flow

flow was in Fig.4. The overall reaction was (1). Silicon tetrachloride vapor reacts with zinc vapor in the vertical furnaces at $1120 \mathrm{~K}-1200 \mathrm{~K}$. Silicon tetrachloride vapor and zinc vapor entered the reaction furnaces at the different direction. Amorphous silicon was the main products. Tail gas which includes zinc, argon and silicon tetrachloride chloride was condensed separately. Zinc chloride was electrolyzed to prepare the zinc. The amorphous silicon particles at the bottom of the reactor then sink to the lower part of the cone by gravity. By heating up solid silicon into molten silicon, molten silicon was condensed into Single crystal silicon rods.

\section{B. Improvements of the Equipment}

Fig.5 is the improvement equipment. The bottom of the Fig. 5 is the key part " $\mathrm{A}$ " of the reactor. Electrical heating was placed in the inlet of Zinc vapor and Silicon tetrachloride to ensure the steam state. Fast reaction rate is feature of this reaction.

The generated silicon of solid crystal (needle, flake and particle shaped) shall accumulate at the bottom part of the vertical reactor, while, the zinc chloride vapor will be discharged from the upper outlet of the reactor, and wait for further treatment. The amorphous silicon particles at the bottom of the reactor then sink to the lower part of the cone by gravity. The lower part, by the help of high frequency coil 1 , is heated up to $1023 \mathrm{~K}$, and impurities of solid zinc and zinc downwards at a preset speed V, allowing for a continuously. 

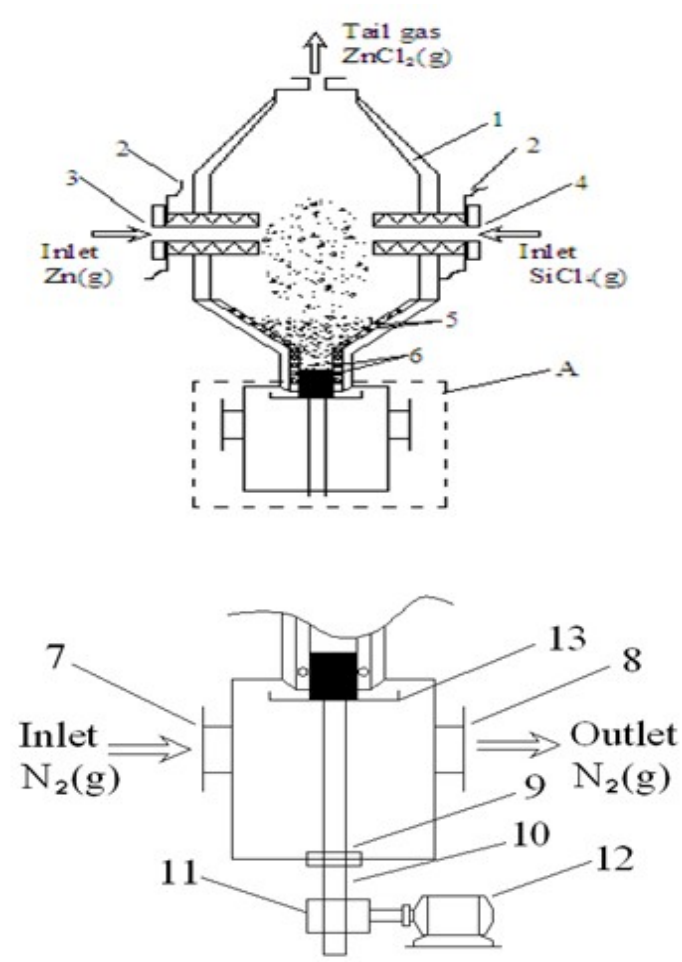

1-Thermal insulating layer 2-electric heater 3-Zinc vapor inlet 4- Silicon tetrachloride vapor inlet 5-high-frequency coil-1 6- high-frequency coil-2 7- Nitrogen inlet 8- Nitrogen outlet 9- gasket ring 10- lifter 11- speed transmission

12-Servo Motor 13-tray A- Single Crystal rod Collection Device

Figure 5. The improved equipment

Chloride in the amorphous silicon are vaporized, and the gaseous zinc continues to react with silicon tetrachloride, and the zinc chloride vapor generated thereof is discharged with the tail gas. Relatively pure amorphous silicon is heated up to $1687 \mathrm{~K}$ by high-frequency coil 2 into molten silicon, which outflows through the reactor's conical bottom spout and is cooled and solidified. The tray of silicon rod moves gradually formation of silicon rods

The key part of the reactor is the silicon single crystal rod collecting device attached to the reactor's lower body. The descending speed $\mathrm{V}$ of the tray is related to the silicon tetrachloride feed rate, and the feed amount of silicon tetrachloride determines the amount of the amorphous silicon to be generated; thus, the amount of amorphous silicon determines the descending speed of the tray. Suppose the conversion rate of silicon tetrachloride and silicon is $80 \%$. For each a mol of $\mathrm{SiCl}_{4}$, the converted amorphous silicon will be $0.8 \mathrm{a} \mathrm{mol}$; if the diameter of the lower end outlet of the reactor is $\mathrm{d} \mathrm{mm}$, then

$$
V=\frac{0.8 a \times M_{S i}}{\rho_{S i} \frac{\pi d^{2}}{4}} .
$$

In (6), $\mathrm{M}_{\mathrm{Si}}$ means molecular weight of $\mathrm{Si}$ and $\rho_{\mathrm{Si}}$ means density of Si. Single crystal silicon rod collecting device in a nitrogen atmosphere can not only prevent the Single crystal silicon rods from being oxidized by air, but also ensure the purity of silicon rods generated. For the natural external environment can cause contaminations to the rods during the manufacturing process, and a nitrogen atmosphere can serve the purpose of avoiding such pollution. What's more, the nitrogen gases may carry off part of the heat within the silicon rods, allow for the molten silicon leaving the reactor to cool down and condense into single crystal silicon rods.

\section{The advantages of the improvement technology}

1) Changing the batch style of zinc reduction of silicon tetrachloride into a continuous production style: The original process is of a batch style, in which it needs to add zinc and collect silicon on a regular basis. By directing continuous zinc vapor and silicon tetrachloride vapor into the reactor, by heating up solid silicon into molten silicon with a high frequency coil at the reactor bottom, the discharging outlet on the reactor bottom is sealed up by the molten and no vapor of zinc or silicon tetrachloride or zinc chloride can escape therefrom; eventually, the new technology achieves a continuous production of zinc reduction of silicon tetrachloride in producing solar grade silicon.

2) Shortening reaction process: In the original process, zinc contained in the amorphous silicon is removed by dissolution after the amorphous silicon is generated and collected, and the purified amorphous silicon is then directed into a single crystal furnace for heating and melting before it is pulled into single-crystal Si rods. As a contrast, the new process directly transforms the silicon amorphous particles into a molten state and makes single crystal silicon rods by pulling.

3) Reducing energy consumption: The adoption of modified Siemens process to produce solar-grade polycrystalline silicon, the Siemens process itself is of a high energy consumption process, not to mention the energy consumed when melting the polycrystalline silicon and then straightening by pulling it to be monocrystalline silicon rods. While, the process of zinc reduction of silicon tetrachloride can realize a continuous production of silicon rods, which can greatly reduce the energy consumption.

\section{CONCLUSION}

The diagrams of $\Delta_{r} G_{m}^{\Theta}$-T and $\kappa_{p}^{\Theta}$-T for the Zinc production reaction were plotted. According to the diagram, the rational temperature range is $1120-1200 \mathrm{~K}$. The thermodynamic analysis was the theoretical basis of technology improvements. The new technology achieved continuous production of zinc reduction of silicon tetrachloride in producing solar grade silicon. The continuous processing, low Energy consumption and the shortening of process flow were the advantage in the improvement of equipment and process. 


\section{REFERENCES}

[1] Norihisa Uesawa, Peng Shen, Susumu Inasawa, Akira Miyoshi, and Yukio Yamaguchi "Kinetic study on gas phase zinc reduction of silicon tetrachloride," Chemical Engineering Journal, vol. 168, pp. 889-895, Febrary 2011.

[2] Z. Luo, X. Cai , R.Y. Hong, L.S. Wang, W.G. Feng,"Preparation of silica nanoparticles using silicon tetrachloride for reinforcement of PU," Chemical Engineering Journal, vol. 187, pp. 357-366, January 2012.

[3] Zhao Chunjiang,Produce of polycrystalline silicon for zinc reduction method, Adevanced meterial industry, May 2009.
[4] Liu SSha and Zhang Jingqiu,An example of calculation and drawing for Gibbs stangrd free energy, Raremetals and cementod carbides. vol. 32, pp. 56-58,Decenber 2004.

[5] Ye Dalun and HU Jianhua,Practical Handbook of Thermodynamic Data for Inorganic Compounds 2ndEd.Beijing:Metallur.gical Industry ,2002.

[6] Xu Baokun and Zhao Muyu, chinese journal of semiconductors, vol. 3 pp. 343-350,May 1982.

[7] Liangyingjiao and Che Yinchang, Handbook of Thermodynamic Data for Inorganic Compounds . Northern University press.

[8] Zhou Hongjun,Zhang Taisheng,Li Hongbo and Chen Mingbo,Study on the industrialized application for zinc reduction of silicon tetrachloride.Adevanced meterial industry, pp.59-62,November 2008 